Check for updates

Cite this: RSC Adv., 2019, 9, 21318

Received 25th April 2019

Accepted 30th June 2019

DOI: $10.1039 / \mathrm{c} 9 \mathrm{ra03070k}$

rsc.li/rsc-advances

\section{In vitro and in vivo evaluation of benzathine foscarnet microcrystals as a potential intravitreal drug depot $\uparrow$}

\author{
Jiaxing Wang, ${ }^{a}$ Yong Tao, ${ }^{\text {b }}$ Jing Feng, ${ }^{\text {b }}$ Yidong Niu, ${ }^{c}$ Jichao Liu ${ }^{c}$ \\ and Yanbin Huang (iD *a
}

\begin{abstract}
Sodium foscarnet is an antiviral drug against cytomegalovirus retinitis, and clinically it is used via frequent intravitreal injection which causes various ocular complications. Here we propose to use benzathine foscarnet in a new salt form with much lower aqueous solubility, and as a potential long-acting intravitreally injectable solid form for foscarnet. Benzathine foscarnet (1:1) microcrystals were synthesized and evaluated both in vitro and in vivo. The aqueous solubility of benzathine foscarnet was $14.2 \mathrm{mM}$, which is in between those of the currently-used sodium foscarnet and our previously-reported calcium foscarnet salt. In a rabbit model, the injected microcrystals last for about 3 weeks in the vitreous, suggesting its solubility and dissolution profile is appropriate for its intended use. However, the injected benzathine foscarnet microcrystals also caused adverse effects in vivo.
\end{abstract}

\section{Introduction}

Foscarnet is one of the few drugs being clinically used for the treatment of cytomegalovirus (CMV) retinitis, which is a common ocular infection for immune-suppressed populations such as AIDS (Acquired Immune Deficiency Syndrome) patients and organ transplant recipients. For those patients without immune recovery, CMV retinitis is still a devastating disease and eventually causes retina damage and blindness. ${ }^{\mathbf{1 , 2}}$

The currently approved form of foscarnet is its sodium salt solution and administered through intravenous infusion. However, due to the existence of the blood-retina barrier, the retina bioavailability of intravenously-infused foscarnet is inadequate for its efficacy. ${ }^{3}$ Consequently, it is a common clinical practice to use the foscarnet sodium solution off-label by direct injection into the vitreous cavity (i.e. intravitreal injection), 1-2 times per week, $2.4 \mathrm{mg}$ in $0.1 \mathrm{~mL}$ saline per injection, and often lasting for the life time of the patients., However, frequent intravitreal injections not only result in low patient compliance, but also cause ocular complications such as cornea damage, endophthalmitis and hemorrhage. ${ }^{5,6}$ A longlasting intravitreal injectable form of foscarnet may significantly reduce the injection frequency and is a clear unmet

\footnotetext{
${ }^{a}$ Key Laboratory of Advanced Materials (MOE), Department of Chemical Engineering, Tsinghua University, Beijing 100084, China. E-mail: yanbin@tsinghua.edu.cn

${ }^{b}$ Department of Ophthalmology, Beijing Chaoyang Hospital, Capital Medical University, Beijing 100020, China.E-mail: drtaoyong@sina.com

${ }^{c}$ Laboratory Animal Unit, Peking University People's Hospital, Beijing 100044, China

† CCDC 1908677. For crystallographic data in CIF or other electronic format see DOI: $10.1039 / \mathrm{c} 9 \mathrm{ra} 03070 \mathrm{k}$
}

clinical need. Chemically, foscarnet has a phosphonoformate structure (Pfa, ${ }^{-}$OOC- $-\mathrm{PO}_{3}{ }^{2-}$ ).

In our previous study, ${ }^{7}$ we evaluated foscarnet calcium microcrystals as a potential intravitreal drug depot. Compared with the highly soluble sodium salt $\left(\mathrm{Na}_{3} \mathrm{Pfa}\right)$, foscarnet calcium $\left(\mathrm{Ca}_{3}(\mathrm{Pfa})_{2}\right)$ has a much lower solubility (the solubility of the sodium and the calcium salts in deionized water are $260 \mathrm{mM}$ and $0.3 \mathrm{mM}$, respectively). After intravitreal injection, the foscarnet calcium microcrystals with initial diameter around 10 $\mu \mathrm{m}$ slowly dissolved, and it was shown it took 3 months for their complete dissolution. Ideally, the gradual dissolution of foscarnet calcium should provide a continuous supply of the active Pfa ions and maintain its intravitreal concentration above the efficacious drug concentration. However, the reported half maximum inhibitory concentrations $\mathrm{EC}_{50}$ for foscarnet against CMVs in the cell culture range from 0.05 to $0.8 \mathrm{mM} .^{8}$ Since the highest drug concentration can be achieved after intravitreal injection of foscarnet calcium microcrystals is its equilibrium solubility $(0.3 \mathrm{mM})$, the solubility of foscarnet calcium, though within the therapeutically relevant range, seems not high enough to maintain the efficacious drug concentration.

The solubility of the drug crystal is a fundamental parameter for a drug depot. It should be low enough to slow down the drug crystal dissolution and last long in the vitreous cavity, while it cannot be too low to achieve the efficacious antiviral concentration. ${ }^{7}$ In this study, we prepared and evaluated, both in vitro and in vivo in a rabbit model, the $N, N^{\prime}$-dibenzyl ethylenediamine (benzathine, Scheme 1) salt of foscarnet as the drug depot solid with aqueous solubility in between that of the sodium and calcium salts. As a hydrophobic amine, benzathine has been clinically used as the cation in Penicillin G Benzathine 


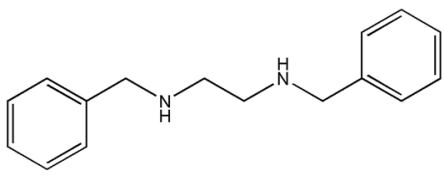

benzathine

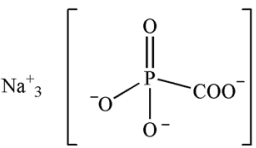

sodium foscarnet
Scheme 1 Molecular structures of benzathine and sodium foscarnet.

(Bicillin L-A®) drug product, which is an intramuscularly injectable suspension of this low solubility penicillin salt to achieve a long lasting therapeutic effect. ${ }^{9}$ The reported median lethal dose $\left(\mathrm{LD}_{50}\right)$ of benzathine in mouse is $388 \mathrm{mg} \mathrm{kg}^{-1}$ and $50 \mathrm{mg} \mathrm{kg}^{-1}$ via the oral and intraperitoneal route, respectively. ${ }^{10}$ However, its ocular use has not been reported, and will be evaluated for the first time in this study.

\section{Methods}

\section{Preparation of benzathine foscarnet}

Benzathine foscarnet microcrystals were synthesized via a simple salt metathesis reaction between foscarnet sodium and $N, N^{\prime}$-dibenzyl ethylenediamine (benzathine) diacetate. In a typical experiment, $1.28 \mathrm{~g}$ ( $4 \mathrm{mmol})$ foscarnet sodium hexahydrate (Hubei Prosperity Galaxy Chemical Co., China) was dissolved in $50 \mathrm{~mL}$ deionized water, and $3.58 \mathrm{~g}$ (10 mmol) $N, N^{\prime}$ dibenzyl ethylenediamine diacetate (Shanghai Sam Chemical Technology Co., China) was dissolved in $60 \mathrm{~mL}$ deionized water. Then the two solutions were mixed and stirred at room temperature for 24 hours. Afterwards, the stirring was stopped and the $\mathrm{pH}$ of the reaction system was measured ( $\mathrm{pH}$ 6.2). The solid was separated by filtration and recrystallized once in the deionized water. After vacuum drying at room temperature for 12 hours, benzathine foscarnet product was collected (yield $41.7 \%)$.

\section{Crystal characterizations}

The benzathine foscarnet product was analyzed with Scanning Electron Microscope (SEM, JSM-7500F, JEOL Ltd., Japan) operated at an accelerating voltage of $3.0 \mathrm{kV}$. Polarized optical microscope (POM, BX41, Olympus) equipped with a digital camera (Moticam Pro 282A, Motic) was also used to observe the morphology of benzathine foscarnet.

Thermogravimetric analysis (TGA) was used to check the existence of water or solvent in crystals and measure the degradation temperature. The sample was placed in an aluminum pan, and characterized using a thermogravimetric analyzer (TA Instruments TGA Q500IR). The samples were heated over the temperature range $30-500{ }^{\circ} \mathrm{C}$ at a constant heating rate of $20{ }^{\circ} \mathrm{C} \mathrm{min}^{-1}$, while purged with a stream of flowing nitrogen at $50 \mathrm{~mL} \mathrm{~min}^{-1}$ throughout the experiment.

Differential Scanning Calorimetry (DSC) tests were performed on a Shimadzu DSC-60 differential scanning calorimeter. 3-5 $\mathrm{mg}$ powder samples were placed in aluminum pans and heated from $30{ }^{\circ} \mathrm{C}$ to $220{ }^{\circ} \mathrm{C}$ at a rate of $10{ }^{\circ} \mathrm{C} \mathrm{min}^{-1}$.
${ }^{1} \mathrm{H}$-nuclear magnetic resonance $\left({ }^{1} \mathrm{H}-\mathrm{NMR}\right)$ and ${ }^{31} \mathrm{P}$-nuclear magnetic resonance $\left({ }^{31} \mathrm{P}-\mathrm{NMR}\right)$ were used to confirm the chemical composition. ${ }^{1} \mathrm{H}$-NMR and ${ }^{31} \mathrm{P}$-NMR tests were performed on a JOEL JNM-ECA600 nuclear magnetic resonance spectrometer. Deuteroxide was used as the solvent. Analysis of the obtained spectra was performed using the MestReNova software.

Inductively coupled plasma optical emission spectrometry (ICP-OES) was used to confirm the chemical composition. Before testing, $100 \mathrm{mg}$ benzathine foscarnet was fully dissolved in $2 \mathrm{~mL}$ nitric acid ( $65 \mathrm{wt} \%)$ and diluted with deionized water to make the final solution volume $25 \mathrm{~mL}$. The concentration of phosphorus was determined using inductively coupled plasma optical emission spectrometry (ICP-OES iCAP6300, Thermo Fisher). The plasma power is $1150 \mathrm{~W}$ and the speed of peristaltic pump is $50 \mathrm{rpm}$. The atomizing flow rate and the auxiliary flow rate were $0.6 \mathrm{~L} \mathrm{~min}^{-1}$ and $0.5 \mathrm{~L} \mathrm{~min}^{-1}$, respectively.

X-ray Photoelectron Spectroscopy (XPS) was also used to confirm the chemical composition. The sample was characterized using a PHI Quantera SXM X-ray photoelectron spectroscopy, by general rules for analysis of GB/T 19500-2004.

Single crystal analysis was conducted to obtain the crystal structure of benzathine foscarnet. Single crystals of benzathine foscarnet were grown in aqueous solutions. A suitable crystal was selected and placed on a SuperNova AtlasS2 diffractometer. The crystal was kept at 100.00 (10) K during data collection. Using Olex2, the structure was solved with the ShelXT structure solution program using direct methods and refined with the ShelXL refinement package using least squares minimization.

X-ray diffraction (XRD) measurement was carried out with a Rigaku D/max-2500 X-ray diffractometer with $\mathrm{Cu} \mathrm{K} \alpha$ radiation (1.54056 ̊). Samples were placed on the sample holder and continuous scans were performed at a speed of $4^{\circ} 2 \theta$ per min and $0.02^{\circ} 2 \theta$ per step in the range $2 \theta=5-40^{\circ}$.

\section{Solubility determination}

Excess benzathine foscarnet were added into $2 \mathrm{~mL}$ different aqueous media: deionized water, $0.9 \% \mathrm{NaCl}$ solutions and $0.1 \mathrm{~mol} \mathrm{~L}^{-1}$ Tris-HCl buffer $(\mathrm{pH}=7)$. The suspension was stirred at $37^{\circ} \mathrm{C}$ at $230 \mathrm{rpm}$ for $48 \mathrm{~h}$. Afterwards, the supernatant was withdrawn and filtered through MF-Millipore Membranes $(220 \mathrm{~nm}) .1 \mathrm{~mL}$ supernatant was mixed with $1 \mathrm{~mL}$ potassium dihydrogen phosphite solution $\left(20 \mathrm{mg} \mathrm{mL}^{-1}\right)$. The concentration of foscarnet in the solution was determined by ${ }^{31} \mathrm{P}-\mathrm{NMR}$, using a JNM-ECA600 600M nuclear magnetic resonance spectrometer. Potassium dihydrogen phosphite was used as an internal calibration standard, whose $\mathrm{P}$ chemical shift in ${ }^{31} \mathrm{P}$ NMR was around $1.6 \mathrm{ppm}$ and $4.2 \mathrm{ppm}$. In contrast, the $\mathrm{P}$ chemical shift of foscarnet was around $0.5 \mathrm{ppm}$ and clearly separated from the phosphite peak, and their peak area ratios can be used to quantitatively calculate the concentration of foscarnet in the solution.

\section{In vivo experiments}

Totally 6 healthy New Zealand white rabbits were used in this study. The Statement for the Use of Animals in Ophthalmic and 
Vision Research (ARVO) was followed, and local institutional approval was received from the review committee of the Beijing Chaoyang Hospital, Capital Medical University. Benzathine foscarnet suspension (100 $\mathrm{mg} \mathrm{mL}^{-1}$ ) was prepared by adding $600 \mathrm{mg}$ benzathine foscarnet into $6 \mathrm{~mL}$ normal saline, and sonicated to make it homogeneous.

Each rabbit was given a single intravitreal injection of $0.2 \mathrm{~mL}$ benzathine foscarnet suspension in one eye and $0.2 \mathrm{~mL}$ saturated solution $(260 \mathrm{mM})$ of foscarnet sodium in another eye. One rabbit was sacrificed with overdose inhalation of general anesthesia (isoflurane) at 2 days after intravitreal injection. Three rabbits were sacrificed at 7 days and other two rabbits were sacrificed at 21 days after intravitreal injection. After extraction of the aqueous humor, the eyeballs of all rabbits were enucleated and the eyes were sectioned behind the lens, vitreous humor was obtained by dissecting it carefully from the retina.

The vitreous samples of all six rabbits were observed under the polarized optical microscope (BX41, Olympus) equipped with a digital camera (Moticam Pro 282A, Motic). To confirm the chemical composition of remained solid, Energy Dispersive Spectroscopy (EDS) was used to characterize these residual crystals under the scanning electron microscope (JSM-7500F).

\section{Results and discussion}

Benzathine foscarnet was synthesized via a simple salt metathesis reaction between foscarnet sodium and benzathine acetate. The product was needle-shaped crystals with length around $100 \mu \mathrm{m}$ and width in the order of 1-10 $\mu \mathrm{m}$ (Fig. 1A and B). Benzathine foscarnet crystals showed a single melting temperature at $168{ }^{\circ} \mathrm{C}$ (Fig. 1D) and apparent degradation occurred above this temperature (Fig. 1C). No weight loss or thermal transition event was observed before the melting temperature, suggesting that the benzathine foscarnet crystal was not a hydrate or solvate.

As a phosphonoformate compound, foscarnet can have up to 3 negative charges with $\mathrm{p} K_{\mathrm{a}}$ values of $0.48,3.41$ and $7.29 .{ }^{11}$ The
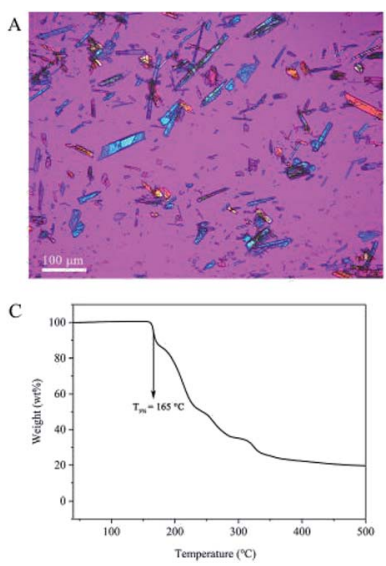
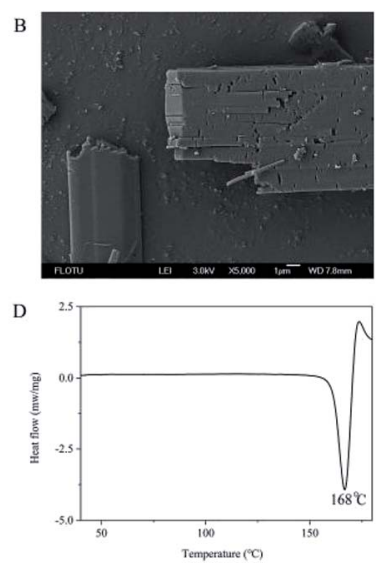

Fig. 1 (A) The POM image (scale bar $100 \mu \mathrm{m}$ ), (B) the SEM image (scale bar $1 \mu \mathrm{m}),(C)$ the TGA thermogram and (D) the DSC thermogram of the benzathine foscarnet microcrystal.

base benzathine (hereafter $\mathrm{B}$ for abbreviation) can have up to 2 positive charges with $\mathrm{p} K_{\mathrm{a}}$ values of 9.39 and $9.99 .^{10}$ Therefore, potentially multiple salt forms can be formed with different ratios between benzathine and foscarnet ions. The phosphorus element percentage in the benzathine foscarnet product was quantified by using ICP and XPS (Table 1), and both the phosphorus percentage value and the $\mathrm{N} / \mathrm{P}$ ratio were consistent with those of a 1:1 benzathine foscarnet salt. This was also consistent with the fact that the reaction solution had a $\mathrm{pH}$ of 6.2 , at which the dominating ion forms in the solution for both benzathine and foscarnet were divalent (i.e., $\mathrm{B}^{2+}$ and $\mathrm{Pfa}^{2-}$ ), making it more likely to form a $1: 1$ salt as $\mathrm{B}^{2+} \mathrm{Pfa}^{2-}$. In terms of the foscarnet content, $1 \mathrm{mg}$ of benzathine foscarnet is equivalent to $0.82 \mathrm{mg}$ of sodium benzathine hexahydrate, the actual drug form used in the current product. Therefore, as a potential foscarnet drug depot, the apparent drug loading of benzathine foscarnet microcrystals is about $82 \%$, much higher than all the drug-encapsulated microsphere products in clinic, whose highest drug loading is $38 \%$ (i.e., Risperdal Consta $\left.{ }^{\circledR}\right) .{ }^{\mathbf{1 2}}$

The ratio between benzathine and foscarnet in the product was further confirmed with single-crystal analysis (Fig. 2). Benzathine foscarnet crystal was determined to be a monoclinic system with one benzathine ion and one foscarnet ion in each unit cell. CCDC 1908677 contains the supplementary crystallographic data for this paper. More crystallography parameters of the benzathine foscarnet crystals are shown in Table 2 .

We then determined the aqueous solubility of benzathine foscarnet using ${ }^{31} \mathrm{P}-\mathrm{NMR}$ with $\mathrm{KH}_{2} \mathrm{PO}_{3}$ as the internal standard (Table 3). In the deionized water, the solubility of benzathine foscarnet was found to be $14.2 \mathrm{mM}$, whose order of magnitude is in between those of calcium foscarnet $(0.3 \mathrm{mM})$ and sodium foscarnet $(260 \mathrm{mM}) .^{7}$ This solubility value $(14.2 \mathrm{mM})$ is also one order of magnitude higher than the upper limit of the reported $\mathrm{EC}_{50}$ range of foscarnet against cytomegalovirus (i.e., $0.8 \mathrm{mM}$ ), and likely to be high enough to maintain the intravitreal drug concentration above the efficacious level. The solubility of benzathine foscarnet in $0.9 \% \mathrm{NaCl}$ solution and $\mathrm{pH} 7.0$ Tris buffer $(0.1 \mathrm{M})$ was even higher (Table 3$)$. The solubility difference among the three foscarnet salts was caused by the different interaction energy between the foscarnet anions and the cations (i.e., sodium, calcium and benzathine cations) in the solid states and in the solution. ${ }^{\mathbf{1 0}}$

Totally 6 rabbits were used in the in vivo evaluation study with two main objectives: one was to see how long it will take for the benzathine foscarnet microcrystals to completely dissolve in the vitreous cavity, and the other was to evaluate their intravitreal toxicity. Each rabbit received an intravitreal injection of

Table 1 Concentration of the P element and the N/P molar ratio of the benzathine foscarnet sample compared with the theoretical result of the $1: 1$ salt

\begin{tabular}{lll}
\hline & Experimental result & Estimation \\
\hline P (wt\%) measured by ICP & 8.9 & 9.0 \\
N/P molar ratio measured by XPS & 2.0 & 2.0
\end{tabular}

N/P molar ratio measured by

$\mathrm{N} / \mathrm{P}$ molar ratio measured by XPS
2.0 


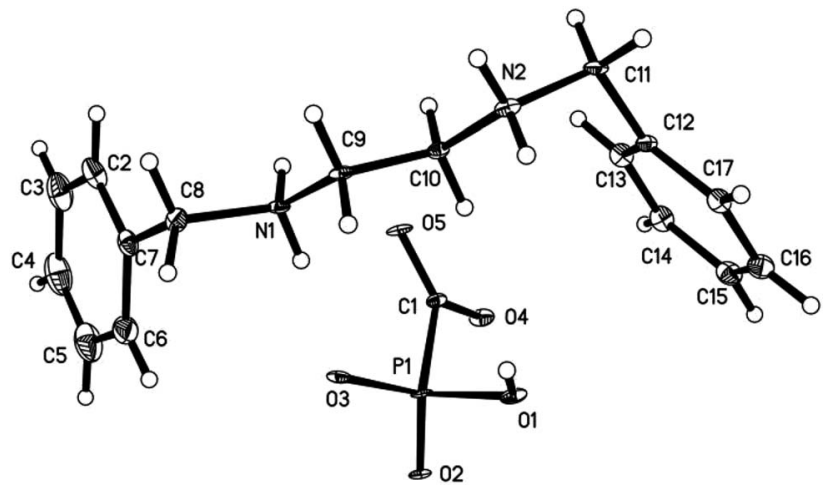

Fig. 2 The ORTEP plot of the crystal structure of benzathine foscarnet. Displacement ellipsoids are drawn at the 30\% probability level.

a $20 \mathrm{mg}$ benzathine foscarnet (equivalent to 16.4 sodium foscarnet hexahydrate) suspended in $0.2 \mathrm{~mL}$ saline in the right eye, and $15.6 \mathrm{mg}$ sodium foscarnet hexahydrate saturated solution in $0.2 \mathrm{~mL}$ saline in the left eye as the control. The animals were sacrificed at certain time points and their vitreous contents were observed under polarized optical microscope. As shown in Fig. 3A, no crystal was observed in any of the sodium foscarnet control eyes, suggesting that the injection of saturated sodium foscarnet solution did not cause crystallization in vivo. On the other hand, needle-shaped crystals were clearly observed in the benzathine foscarnet-injected eye, from day 2 and up to day 21 (Fig. 3B-D). Energy Dispersive Spectroscopy (EDS) was used to characterize these residual crystals under the scanning electron microscope, and both phosphorus and nitrogen
Table 3 Solubility of benzathine foscarnet in the deionized water, $0.9 \%$ sodium chloride solution and Tris- $\mathrm{HCl}$ buffer $(\mathrm{pH} 7)$ compared with solubility values of foscarnet calcium and the foscarnet sodium in the literature ${ }^{7}$

\begin{tabular}{lcll}
\hline Solubility $(\mathrm{mM})$ & $\mathrm{H}_{2} \mathrm{O}$ & $\begin{array}{l}0.9 \% \\
\mathrm{NaCl}\end{array}$ & $\begin{array}{l}\text { Tris-HCl } \\
\text { buffer }(\mathrm{pH} 7,0.1 \mathrm{M})\end{array}$ \\
\hline Benzathine foscarnet & 14.2 & 25.0 & 37.5 \\
$\mathrm{Ca}_{3}(\mathrm{Pfa})_{2}$ & 0.3 & - & - \\
$\mathrm{Na}_{3} \mathrm{Pfa}$ & 260 & - & -
\end{tabular}

elements were observed (data not shown), confirming that these were benzathine foscarnet crystals.

On the toxicity side, inflammation (i.e., conjunctival congestion and pupil shrinkage) was observed at day 2 in all the animal eyes of both the benzathine foscarnet and the sodium foscarnet groups (Fig. 4A and B). At day 7, the inflammation level was reduced (Fig. 4C and D). Throughout the study period up to 21 days, the vitreous in the sodium foscarnet control group remained clear (Fig. 4E). However, in the benzathine foscarnet group, the vitreous was opaque, the light reflex disappeared, and cataract was observed (Fig. 4E). The adverse effect of the sodium foscarnet group was suspected to be due to the high foscarnet concentration of the injected solution (equivalent to $78 \mathrm{mg} \mathrm{mL} \mathrm{m}^{-1}$ sodium foscarnet hexahydrate $v s$. normally used $24 \mathrm{mg} \mathrm{mL}^{-1}$ ). On the other hand, benzathine foscarnet microcrystals should gradually dissolve in the vitreous, and the foscarnet concentration in vivo should be lower than that of the sodium foscarnet group at each time point. The fact that the adverse effect was more severe in the

Table 2 Crystallography data of the benzathine foscarnet crystal

\begin{tabular}{|c|c|}
\hline Empirical formula & $\mathrm{C}_{17} \mathrm{H}_{23} \mathrm{~N}_{2} \mathrm{O}_{5} \mathrm{P}$ \\
\hline Crystal system & Monoclinic \\
\hline$a(\AA)$ & $6.7932(3)$ \\
\hline$b(\AA)$ & $10.6086(5)$ \\
\hline$c(\AA)$ & $24.6911(12)$ \\
\hline$\gamma\left({ }^{\circ}\right)$ & 90 \\
\hline Volume $\left(\AA^{3}\right)$ & $1777.18(14)$ \\
\hline$Z$ & 4 \\
\hline$\rho_{\text {calc }}\left(\mathrm{g} \mathrm{cm}^{-3}\right)$ & 1.369 \\
\hline$\mu\left(\mathrm{mm}^{-1}\right)$ & 1.641 \\
\hline$F(000)$ & 776.0 \\
\hline Independent reflections & $3464\left[R_{\text {sigma }}=0.0524\right]$ \\
\hline Data/restraints/parameters & $3464 / 132 / 228$ \\
\hline Goodness-of-fit on $F^{2}$ & 1.286 \\
\hline Final $R$ indexes $[I \geq 2 \sigma(I)]$ & $R_{1}=0.1069, \mathrm{w} R_{2}=0.2699$ \\
\hline Final $R$ indexes [all data] & $R_{1}=0.1145, \mathrm{w} R_{2}=0.2728$ \\
\hline Largest diff. peak/hole $\left(\mathrm{e} \AA^{-3}\right)$ & $0.75 /-0.68$ \\
\hline
\end{tabular}



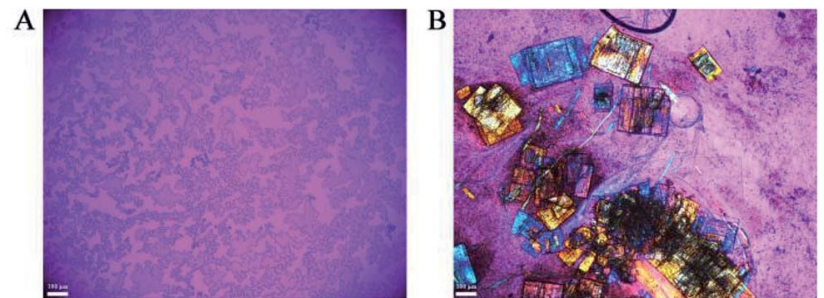

C
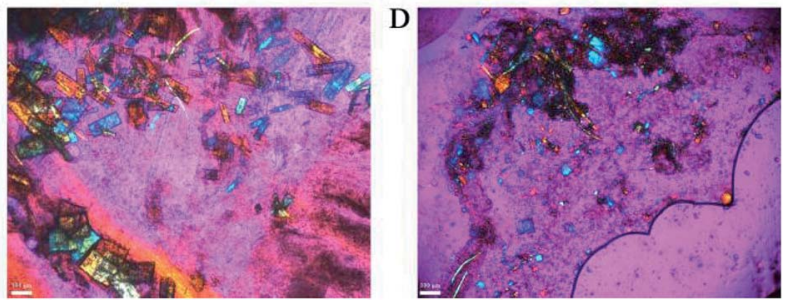

Fig. 3 The POM images (scale bar $100 \mu \mathrm{m}$ ) of the vitreous samples injected with foscarnet sodium (A) and with benzathine foscarnet at day 2 (B), day 7 (C) and day 21 (D) after injection.
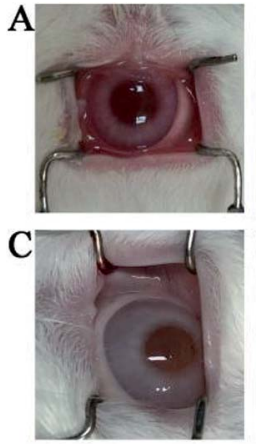

$\mathrm{E}$

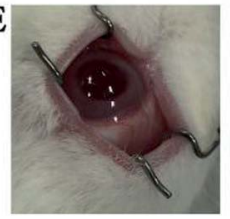

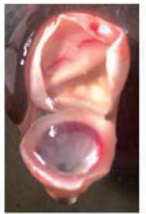
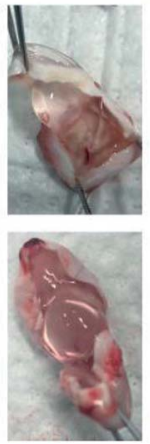
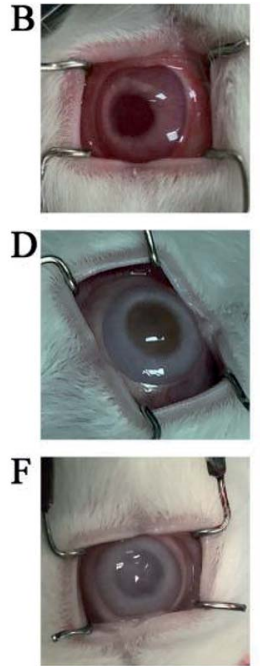
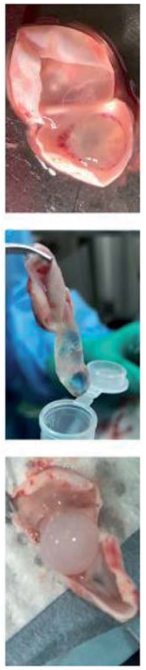

Fig. 4 Pictures of rabbit eyes after injection of foscarnet sodium at day $2(A)$, day $7(C)$, day $21(E)$, and pictures of rabbit eyes after injection of benzathine foscarnet at day 2 (B), day 7 (D) day 21 (F).

benzathine foscarnet group may be due to the toxicity of benzathine component, whose toxicity profile in the ocular has not been previously reported in the literature.

\section{Conclusions}

In summary, we have synthesized the benzathine foscarnet $(1: 1)$ microcrystal, and evaluated it as a potential drug depot for the intravitreally injectable form of the anti-viral drug foscarnet. The aqueous solubility of benzathine foscarnet was found in between the previously-studied sodium and the calcium salts of foscarnet. The preliminary in vivo tests in a rabbit model successfully demonstrated that the injected benzathine foscarnet crystals last for 3 weeks in the vitreous. However, the benzathine component in the salt, which has been used in the intravenous route but never been tested in the ocular route, caused significant adverse effect in the rabbit study. Therefore, we have demonstrated that foscarnet salts with lower solubility is indeed a valid approach to identify candidates for foscarnet drug depot, but other bases may need to be screened to achieve a balanced combination of solubility and safety profile.

\section{Conflicts of interest}

There are no conflicts to declare.

\section{Acknowledgements}

This work was supported by the Beijing Chaoyang 1351 Talent Training Program (No. CYXX-2017-21) and the Natural Science Foundation of China (No. 21434008).

\section{References}

1 A. D. Port, A. Orlin, S. Kiss, S. Patel, D. J. D'Amico and M. P. Gupta, J. Ocul. Pharmacol. Ther., 2017, 33, 224.

2 N. Ford, Z. Shubber, P. Saranchuk, S. Pathai, N. Durier, D. P. O'brien, E. J. Mills, F. Pascual, E. Hoen, G. N. Holland and D. Heiden, Clin. Infect. Dis., 2013, 57, 1351. 3 S. C. She, G. A. Peyman and J. A. Schulman, Int. Ophthalmol., 1988, 12, 151.

4 M. Diaz-Llopis, E. España, G. Muñoz, A. Navea, E. Chipont, J. Cano, J. L. Menezo and F. J. Romero, Br. J. Ophthalmol., 1994, 78, 120.

5 R. D. Jager, L. P. Aiello, S. C. Patel and E. T. Cunningham, Retina, 2004, 24, 676.

6 G. A. Peyman, E. M. Lad and D. M. Moshfeghi, Retina, 2009, 29, 875.

7 Y. Sun, Y. Tao, Q. Cao and Y. Huang, Chem. Commun., 2017, 53, 5139.

8 Foscavir product label, https:/www.drugs.com/pro/ foscavir.html.

9 Bicillin L-A product label, https://www.drugs.com/pro/ bicillin-la.html.

10 P. H. Stahl and C. G. Wermuth, Handbook of Pharmaceutical Salts: Properties, Selection and Use. Wiley-VCH, 2002, p. 312.

11 S. Warren and M. R. Williams, J. Chem. Soc. B, 1971, 4, 618.

12 Risperdal Consta product label, https://www.drugs.com/ risperdal_consta.html. 